EPJ Web of Conferences 81, 01017 (2014)

DOI: 10.1051 epjconf/ 20148101017

(C) Owned by the authors, published by EDP Sciences, 2014

\title{
Kaonic atoms - studies of the strong interaction with strangeness
}

J. Marton ${ }^{1, a}$, M. Bazzi ${ }^{2}$, G. Beer ${ }^{3}$, C. Berucci ${ }^{1,2}$, A.M. Bragadireanu ${ }^{1,5}$, M. Cargnelli ${ }^{1}$, C. Curceanu2 ${ }^{2}$, A. d'Uffizi ${ }^{2}$, C. Fiorini ${ }^{4}$, F. Ghio ${ }^{6}$, C. Guaraldo ${ }^{2}$, R. Hayano ${ }^{7}$, M. Iliescu², T. Ishiwatari ${ }^{1}$, M. Iwasaki ${ }^{8}$, P. Levi Sandri ${ }^{2}$, S. Okada ${ }^{8}$, D. Pietreanu ${ }^{2,5}$, T. Ponta ${ }^{5}$, R. Quaglia ${ }^{4}$, A. Romero Vidal ${ }^{10}$, E. Sbardella ${ }^{2}$, A. Scordo ${ }^{2}$, H. Shi ${ }^{1,7}$, D.L. Sirghi ${ }^{2,5}$, F. Sirghi ${ }^{2,5}$, H. Tatsuno ${ }^{2}$, O. Vazquez Doce ${ }^{9}$, E. Widmann ${ }^{1}$, B. Wünschek ${ }^{1}$, and J. Zmeskal ${ }^{1}$

${ }^{1}$ Stefan-Meyer-Institut für subatomare Physik, Boltzmanngasse 3, 1090 Wien, Austria

${ }^{2}$ INFN, Laboratori Nazionali di Frascati, C.P. 13, Via E. Fermi 40, I-00044 Frascati (Roma), Italy

${ }^{3}$ Dep. of Physics and Astronomy, University of Victoria, P.O.Box 3055, Victoria B.C. Canada V8W3P6

${ }^{4}$ Politecnico di Milano, Dip. di Elettronica e Informazione, Piazza L. da Vinci 32, I-20133 Milano, Italy

${ }^{5}$ Institutul National pentru Fizica si Inginerie Nucleara Horia Hulubei, Reactorului 30, Magurele, Romania

${ }^{6}$ INFN Sez. di Roma I and Instituto Superiore di Sanita I-00161, Roma, Italy

${ }^{7}$ University of Tokyo,7-3-1, Hongo, Bunkyo-ku,Tokyo, Japan

${ }^{8}$ RIKEN, Institute of Physical and Chemical Research, 2-1 Hirosawa, Wako, Saitama 351-0198 Japan

${ }^{9}$ Excellence Cluster Universe, Tech. Univ. München, Boltzmannstraße 2, D-85748 Garching, Germany

${ }^{10}$ Universidade de Santiago de Compostela, Casas Reais 8, 15782 Santiago de Compostela, Spain

\begin{abstract}
The strong interaction of charged antikaons $\left(\mathrm{K}^{-}\right)$with nucleons and nuclei in the low-energy regime is a fascinating topic. The antikaon plays a peculiar role in hadron physics due to the strong attraction antikaon-nucleon which is a key question for possible kaonic nuclear bound states. A rather direct experimental access to the antikaonnucleon scattering lengths is provided by precision X-ray spectroscopy of transitions to low-lying states in light kaonic atoms like kaonic hydrogen and deuterium. After the successful completion of precision measurements on kaonic hydrogen and helium isotopes by SIDDHARTA at DA $\Phi N E / L N F$, new X-ray studies with the focus on kaonic deuterium are in preparation (SIDDHARTA2). In the future with kaonic deuterium data the antikaon-nucleon isospin-dependent scattering lengths can be extracted for the first time. An overview of the experimental results of SIDDHARTA and an outlook to future perspectives in the SIDDHARTA2 experiments in this frontier research field will be given.
\end{abstract}

\section{Introduction}

Hadronic atoms are belonging to the group of exotic atoms in which instead of an electron a heavier particle is bound in the atom. In the case of hadronic atoms pionic and kaonic atoms were studied for a wide range of elements (see reviews [1]). In these systems the low-energy strong interaction of Goldstone bosons with nucleons and nuclei can be studied experimentally with high accuracy.

a e-mail: johann.marton@oeaw.ac.at 
Especially the lightest hadronic atoms (i.e. hadronic hydrogen and helium isotopes) are of great interest for understanding the fundamental strong interaction in the framework of modern theory.

With light kaonic atoms like kaonic hydrogen/deuterium one is able to study the low-energy strong interaction involving the strange quark. Due to the mass of the strange quark (more than one orderof-magnitude heavier than the up and down quarks but much lighter than the next quark generations) it provides access to spontaneous and explicit symmetry breaking related to the mass generation.

There are several sources of information about the low-energy strong interaction with strangeness: Scattering data in elastic, charge exchange and hyperonic channels, branching ratios of inelastic processes $\left(\mathrm{K}^{-} \mathrm{p}\right.$ capture) and last-but-not-least data from the X-ray spectra of kaonic atoms (hadronic shift and width).

In contrast to kaon scattering data and branching ratios (no new data are available) new and precise data on X-ray transitions in light kaonic atoms were obtained by the SIDDHARTA experiment recently. The availability of high quality $\mathrm{K}^{-}$sources like DAФNE/LNF and high resolution solid state X-ray detectors lead to a remarkable progress in the study of light kaonic atoms. The electronpositron collider DAФNE plays a crucial role as a world-wide unique source of nearly mono-energetic low-energy $\mathrm{K}^{-}$from the $\Phi$ vector meson decay.

\section{Lightest kaonic atoms}

The measurement and results of experiments on the lightest kaonic atoms are presented in the following.

\subsection{Measurement of X-ray transitions}

The lightest hadronic atoms with strangeness are kaonic hydrogen $\left(\mathrm{K}^{-} \mathrm{p}\right)$, kaonic deuterium $\left(\mathrm{K}^{-} \mathrm{d}\right)$ and kaonic helium isotopes $\left({ }^{3} \mathrm{He},{ }^{4} \mathrm{He}\right)$ in which one can study the antikaon $\left(\mathrm{K}^{-}\right)$-nucleon interaction in an bound state, i.e. at lowest energies, in a fairly direct way. A big advantage is the rather direct access to the strong interaction observables - the shift $\epsilon$ and width $\Gamma$ of low-lying states.

The experimental method used is precision X-ray spectroscopy of the K transitions (Lyman series) np-1s (kaonic hydrogen) or L transitions (Balmer series) nd-2p (kaonic helium) X-ray transitions respectively. The shift is given by the deviation from the pure electromagnetic transition energy which can be calculated with sufficient precision. It has to be noted that only the lowest quantum states are showing measurable effects caused by strong interaction (e.g. ground state $1 \mathrm{~s}$ in the case of kaonic hydrogen).

\subsection{Experimental data on $\mathrm{K}^{-} \mathrm{p}$ interaction}

\subsubsection{SIDDHARTA experiment}

In the SIDDHARTA experiment at DAФNE/LNF the X-ray transitions in kaonic hydrogen and helium atoms were measured with a cylinder-shaped array of 144 Silicon Drift Detectors (SDDs) [2] surrounding a cryogenic gas target with pure hydrogen or helium fillings. These SDDs have an active area of $100 \mathrm{~mm}^{2}$ providing a large solid angle, high intrinsic efficiency in the region of interest, very good energy resolution $(\Delta \mathrm{E} \sim 150 \mathrm{eV}$ at $5.9 \mathrm{keV})$ and timing capability. Especially important is the timing capability of SDDs in order to overcome a limitation of the former DEAR experiment which suffered from the high asynchronous background. With the time correlation between the incoming $\mathrm{K}^{-}$ and the emitted X-ray the asynchronous background can be suppressed by orders of magnitude. The layout and detailed information of the experiment can be found in [3]. 


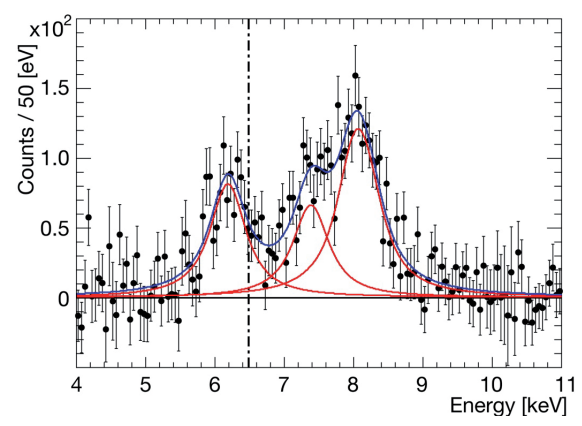

Figure 1. Measured X-ray spectrum of kaonic hydrogen with background components removed. The calculated e.m. position of the $\mathrm{K}_{\alpha}$ line is indicated by a dashed line.

\subsubsection{X-ray spectrum}

The SIDDHARTA experiment [4] measured the X-ray spectrum of kaonic hydrogen in a gaseous target (see Fig. 1). We could also deduce the yields for $\mathrm{K}_{\alpha}=0.012_{-0.004}^{+0.003}$ and the yield for all $\mathrm{K}$ transitions $\mathrm{K}_{t o t}=0.045_{-0.012}^{+0.009}$ [5] which is very important for the theory of the electromagnetic cascade.

The deviation of the measure transition energy $\mathrm{E}_{n p \rightarrow 1 s}^{\text {meas. }}$ from the pure electromagnetic transition energy $\mathrm{E}_{n p \rightarrow 1 s}^{c a l c .}$ of the Lyman lines and the line broadening due to strong interaction was determined.

$$
\epsilon_{1 s}=E_{n p \rightarrow 1 s}^{\text {meas. }}-E_{n p \rightarrow 1 s}^{\text {calc. }}
$$

A comparison of the results of recent experiments on kaonic hydrogen is given in the Fig. 2.

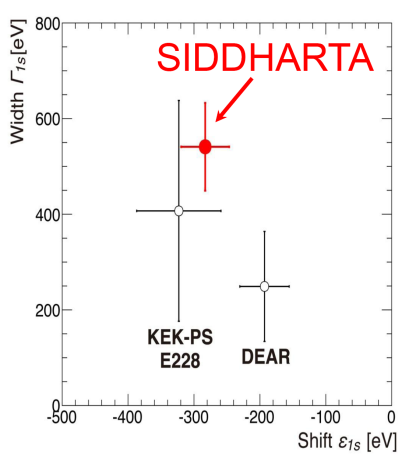

Figure 2. Hadronic shift and width from recent X-ray experiments on kaonic hydrogen. KEK-PS E228 [6], DEAR [7] and SIDDHARTA [3]. One can see that the SIDDHARTA delivered the most precise results up-to-now.

\subsection{Open issue: Kaonic deuterium}

In spite of the big success of SIDDHARTA some goals of new sophisticated experiments are still to be met. Top priority has the measurement of the shift and width of the ground state of kaonic deuterium providing the most stringent constraint on the isospin-dependent antikaon-nucleon interaction. This information is heavily demanded by theory to test the current description in the framework of effective field theory. 


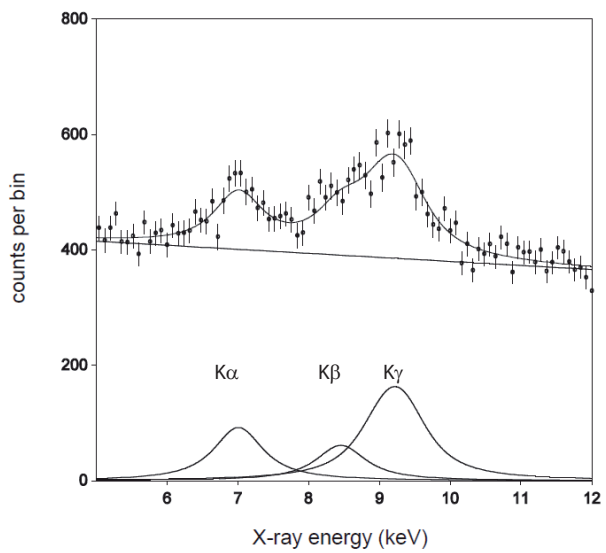

Figure 3. Monte Carlo simulation of the kaonic deuterium X-ray spectrum [8] in a realistic SIDDHARTA2 setup at DAФNE. Here as input parameters $\epsilon_{1 s}=-800 \mathrm{eV}$ and $\Gamma_{1 s}=800 \mathrm{eV}$ and an integrated luminosity of 600 $\mathrm{pb}^{-1}$ were used. The signal-to-background ratio $\mathrm{K} \alpha$ to background signals amounts to $1: 2.7$.

In a first attempt the X-ray spectrum with a pure deuterium filling was measured by SIDDHARTA. We could not deduce the shift and width values but we obtained an upper limit for the K X-ray yield. The total yield was found to be $<0.0143$, the yield of $\mathrm{K} \alpha<0.0039$ [9].

For the SIDDHARTA2 experiment a strongly improved apparatus was designed and is in preparation: Optimized geometry and target density, $\mathrm{K}^{+}$discrimination, active shielding, operation of SDDs at lower temperature for improved timing performance. The expected X-ray energy spectrum according to Monte Carlo study is shown in Fig. 3.

\section{Antikaon-nucleon scattering lengths}

The precise determination of the strong interaction parameters is essential to determine the $\mathrm{K}^{-}-\mathrm{p}$ scattering length $\mathrm{a}_{p}$. For the extraction of $\mathrm{a}_{p}$ the Deser-Trueman formula [10] was used:

$$
\epsilon_{1 s}+\frac{i}{2} \Gamma_{1 s}=2 \alpha^{3} \mu_{c}^{2} a_{p}
$$

where $\alpha$ is the fine structure constant and $\mu_{c}$ the reduced mass.

This simple formula 2 had to be refined in order to take into account Coulomb corrections. An improved Deser-type formula was accordingly developed [11]:

$$
\epsilon_{1 s}+\frac{i}{2} \Gamma_{1 s}=2 \alpha^{3} \mu_{c}^{2} a_{p}\left(1-2 \alpha \mu_{c}(\ln \alpha-1) a_{p}\right)
$$

from the knowledge of $a_{p}$ one gets the sum of isospin $0 a_{0}$ and isospin $1 a_{0}$ scattering lengths:

$$
a_{p}=a_{0}+a_{1} .
$$

In order to extract the isospin scattering lengths $a_{0}$ and $a_{1}$ the values of hadronic shift and width of kaonic deuterium are mandatory giving the information on the $\mathrm{K}^{-} \mathrm{n}$ scattering length which is related to $\mathrm{a}_{1}$.

$$
K^{-} n=a_{1} .
$$


MESON 2014 $-13^{\text {th }}$ International Workshop on Production, Properties and Interaction of Mesons

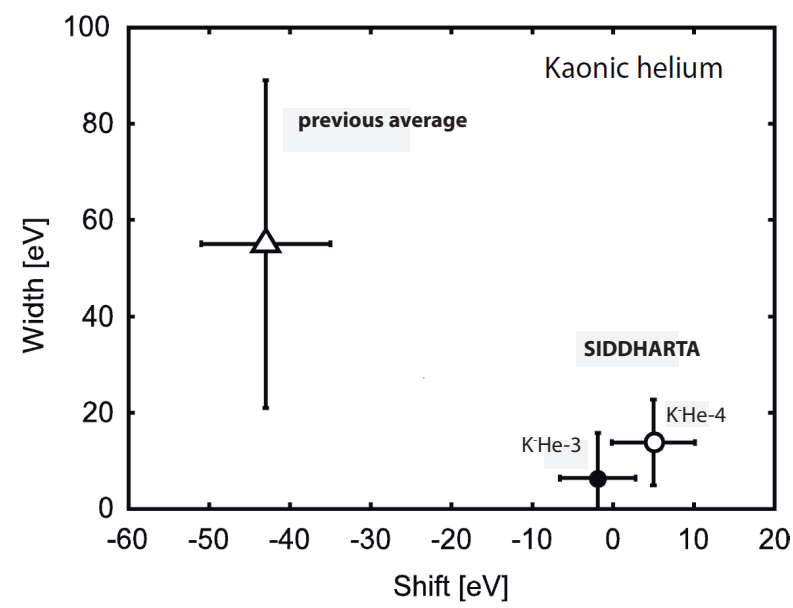

Figure 4. Results on the shift $\epsilon_{2 p}$ and width $\Gamma_{2 p}$ of the $2 \mathrm{p}$ level in kaonic helium obtained in X-ray experiments. The old experiments $[13,14]$ revealed an average of $\epsilon_{2 p}=43 \pm 8 \mathrm{eV}$ for kaonic ${ }^{4} \mathrm{He}$ whereas the results of the recent SIDDHARTA experiments [15] give a small shift in agreement with theory.

From the kaonic deuterium data using equ.3 one can determine analog to kaonic hydrogen $\mathrm{a}_{K^{-}} d$. and subsequently with equ. 4 the isospin scattering lengths $\mathrm{a}_{0}$ and $\mathrm{a}_{1}$.

$$
\begin{gathered}
a_{K^{-} d}=\frac{4\left[m_{N}+m_{K}\right]}{2\left[m_{N}+m_{K}\right]} Q+C, \\
Q=\frac{1}{2}\left[a_{K^{-} p}+a_{K^{-} n}\right]=\frac{1}{4}\left[a_{0}+3 a_{1}\right] .
\end{gathered}
$$

However, several corrections like multiple scattering have to be considered which are still matter of theoretical studies [12].

\subsection{Kaonic helium isotopes}

The study of kaonic helium isotopes opens the access to the interaction of antikaons with simple nuclei. In kaonic helium the Balmer X-ray transtions (e.g. $3 d \rightarrow 2 p$ ) are in a similar energy range like the kaonic hydrogen Lyman lines. The big advantage of kaonic helium is the much higher X-ray yield compared to kaonic hydrogen. Therefore, in SIDDHARTA the measurement of kaonic helium-4 was also used as check of the experiment but has also high importance for the understanding of the physics involved. SIDDHARTA also succeeded to measure the $2 p$ state shift $\epsilon_{2 p}$ [15] and width $\Gamma_{2 p}$ [16] of kaonic He-4 and He-3 (first time measurement of kaonic helium-3 X-ray spectrum). The large shift found in old experiments can be ruled out (see Fig. 4). However, theoretical studies yielded a possible $2 \mathrm{p}$ state shift of up to $\sim 10 \mathrm{eV}$ and a isotopic difference between $\mathrm{K}^{-} \mathrm{He}-3$ and $\mathrm{K}^{-} \mathrm{He}-4$ [17] under the assumption of the existence of kaonic nuclear bound states. A possible (small) isotopic difference between kaonic He-3 and He-4 cannot be ruled out by the SIDDHARTA data. Additionally the X-ray yields of the L transitions in kaonic helium were extracted [18] from the SIDDHARTA data which are valuable input for theoretical cascade models.

\section{Outlook to new experiments}

The SIDDHARTA experiment at DAФNE finished the kaonic hydrogen data analysis recently and obtained values of the low-energy strong interaction parameters of kaonic hydrogen with unprecedented 
accuracy. Therefore, crucially improved constraints for the theory are available now. The impact of SIDDHARTA on the theoretical work on the $\mathrm{K}^{-}$-nucleon interaction at threshold is remarkable. Subsequently a consistent picture was achieved using effective field theory with coupled channels and putting together various sources of information on the antikaon-nucleon interaction [19]. In spite of the big success of SIDDHARTA an extremely important information is still open - the case of kaonic deuterium has the highest priority for the SIDDHARTA follow-up experiment SIDDHARTA-2.

\section{Acknowledgements}

We thank C. Capoccia, G. Corradi, B. Dulach, and D. Tagnani from LNF-INFN; and H. Schneider, L. Stohwasser, and D. Stückler from Stefan-Meyer-Institut, for their fundamental contribution in designing and building the SIDDHARTA setup. We thank as well the DAФNE staff for the excellent working conditions and permanent support. Part of this work was supported by the European Community- Research Infrastructure Integrating Activity "Study of Strongly Interacting Matter" (HadronPhysics2, Grant Agreement No. 227431, and HadronPhysics3 (HP3) Contract No. 283286) under the Seventh Framework Programme of EU; HadronPhysics I3 FP6 European Community program, Contract No. RII3-CT-2004- 506078; Austrian Science Fund (FWF) (P24756-N20); Austrian Federal Ministry of Science and Research BMBWK 650962/0001 VI/2/2009; Romanian National Authority for Scientific Research, Contract No. 2-CeX 06-11-11/2006; and the Grant-in-Aid for Specially Promoted Research (20002003), MEXT, Japan.

\section{References}

[1] E. Friedman, A. Gal, Phys. Rep. 452, 89 (2007), C.J. Batty, E. Friedman, A. Gal, Phys. Rep. 287, 385 (1997).

[2] M. Bazzi, et al., Nuclear Instruments and Methods in Physics Research Section A 628, 264 (2011).

[3] M. Bazzi et al., Physics Letters B 704, 113 (2011), Bazzi, M., Beer, G., Bombelli, L., Bragadireanu, A. M., Cargnelli, M. et al. (SIDDHARTA collaboration), Nuclear Physics A 881, 88 (2012).

[4] C. Curceanu et al., EPJ Web of Conferences 66, 09004 (2014).

[5] H. Shi et al., EPJ Web of Conferences 66, 09016 (2014).

[6] M. Iwasaki et al., Phys. Rev. Lett. 78, 3097(1997), T. M. Ito, et al., Phys. Rev. C 58, 2366 (1998).

[7] G. Beer et al., Phys. Rev. Lett. 94, 212302 (2005).

[8] M. Cargnelli, private communication.

[9] M. Bazzi [SIDDHARTA], Nucl. Phys. A 907, 69 (2013).

[10] S. Deser et al., Phys. Rev. 96, 774 (1954), T.L. Trueman, Nucl. Phys. 26, 57 (1961).

[11] U.-G. Meißner, U. Raha, A. Rusetsky, Eur. Phys. J. C 35, 349 (2004).

[12] U.-G. Meißner, U. Raha, A. Rusetsky, Eur. Phys. J. C 47, 473 (2006).

[13] S. Baird, et al., Nucl. Phys. A 392, 297 (1983).

[14] C.J. Batty, Nucl. Phys. A 508, 89 (1990).

[15] M. Bazzi, et al., Phys. Lett. B 681, 310 (2009).

[16] M. Bazzi et al., Physics Letters B 714, 40 (2012).

[17] Y. Akaishi, Proc. of EXA2005.

[18] M. Bazzi et al., Eur.Phys.J. A 50, 91 (2014).

[19] Y. Ikeda, T. Hyodo and W. Weise, Physics Letters B 706, 63 (2011). 